\title{
Reflets
}

Revue d'intervention sociale et communautaire

\section{Le militantisme dans le champ de l'intervention sociale et communautaire}

\author{
Simon Lapierre, Patrick Ladouceur, Vanessa Couturier, Isabelle Côté et David \\ Buetti
}

Volume 23, numéro 1, printemps 2017

URI : https://id.erudit.org/iderudit/1040746ar

DOI : https://doi.org/10.7202/1040746ar

Aller au sommaire du numéro

Éditeur(s)

Reflets, Revue d'intervention sociale et communautaire

ISSN

1203-4576 (imprimé)

1712-8498 (numérique)

Découvrir la revue

Citer ce document

Lapierre, S., Ladouceur, P., Couturier, V., Côté, I. \& Buetti, D. (2017). Le

militantisme dans le champ de l'intervention sociale et communautaire.

Reflets, 23(1), 10-14. https://doi.org/10.7202/1040746ar d'utilisation que vous pouvez consulter en ligne. 


\section{Le militantisme dans le champ de l'intervention sociale et communautaire}

Simon Lapierre, Ph. D., professeur agrégé

École de service social, Université d'Ottawa

Patrick Ladouceur, candidat à la maîtrise

École de service social, Université d'Ottawa

Vanessa Couturier, coordonnatrice

Collectif de recherche féministe anti-violence (FemAnVi)

Université d'Ottawa

Isabelle Côté, Ph. D., stagiaire postdoctorale

Institut de recherches et d'études féministes (IREF)

David Buetti, candidat au doctorat

École interdisciplinaire des sciences de la santé, Université d'Ottawa

"Je crois qu'il est important de militer parce que c'est une façon de redonner à la société et de se considérer non pas comme une personne ayant accompli quelque chose de manière individuelle, mais plutôt comme faisant partie d'un mouvement qui s'inscrit dans l'histoire."

(Angela Davis, 1997, traduction libre)

Au cours des dernières décennies, plusieurs mouvements ont marqué positivement l'histoire, contribuant à la création d'une société plus juste et plus égalitaire. Ces changements sont le résultat des efforts acharnés de militantes et militants. Pour certains groupes, militer s'est avéré et s'avère toujours une stratégie essentielle pour assurer le respect de leurs droits et parfois même leur survie. Pensons, par exemple, au mouvement des droits civiques aux États-Unis et autres initiatives anti-racistes, aux luttes pour la reconnaissance des communautés autochtones et le respect de leurs droits, au mouvement féministe, au mouvement LGBTTQ* et aux mouvements pour la défense des droits des 
personnes en situation de handicap ou ayant des problèmes de santé mentale. Au Canada, les communautés francophones vivant en situation minoritaire ont aussi dû lutter pour faire reconnaître leur existence et pour faire respecter leurs droits (Bock, 2010).

Si la contribution essentielle du militantisme au changement social est indéniable, quelle est son importance dans le champ du travail social? Le militantisme constitue-t-il une forme d'intervention sociale ou communautaire? Dans un contexte où le travail social est souvent réduit à la dimension de l'intervention individuelle, tant dans ses représentations que dans ses applications, le militantisme n'est pas nécessairement perçu comme une stratégie d'intervention pertinente ou légitime. L'individualisation des problèmes sociaux et la professionnalisation de l'intervention sociale sont aussi des tendances qui contribuent à la marginalisation du militantisme dans la formation et dans la pratique du travail social (Lapierre et Levesque, 2013). De plus, certaines contraintes organisationnelles peuvent limiter le recours à des actions collectives et militantes, particulièrement dans le secteur institutionnel (Mullaly, 2007; Palumbo et Friedman, 2014).

Néanmoins, l'histoire du travail social démontre qu'il y a toujours eu des intervenantes et intervenants sociaux engagés dans une démarche visant davantage le changement social que le changement individuel ou le contrôle social. Cette vision de l'intervention sociale, qui s'appuie sur des principes de justice sociale, d'égalité et de solidarité, tire ses origines du Settlement House Movement, qui s'est développé en Angleterre et aux États-Unis à la fin du XIX et au début du XX ${ }^{\mathrm{e}}$ siècle (St-Amand, 2010). Jane Addams, fondatrice de la Hull-House à Chicago et pionnière dans le domaine du travail social, a été reconnue comme une militante pour la paix ainsi que pour les droits des femmes et des communautés immigrantes.

$\mathrm{Au}$ cours des dernières décennies, certains milieux d'intervention ont davantage favorisé la mise en place d'actions collectives et militantes. C'est le cas, notamment, des organismes du secteur communautaire privilégiant des approches féministes et structurelles (Boisclair, et collab., 2010; Mullaly, 2007).

Les individus, les groupes et les communautés avec lesquels les intervenantes et intervenants sociaux sont en contact sont souvent aux prises avec des conditions de vie difficiles et injustes, qui sont généralement liées à une situation d'oppression (PullenSansfaçon, 2013). Face à ce constat, nous estimons que les interventions sociales et communautaires devraient viser des améliorations durables dans les conditions de vie, tout en luttant contre les oppressions. Le travail social devrait donc avoir une visée de changement social. Cela exige des interventions et des actions collectives qui s'inscrivent dans un mouvement plus large. 
Ce premier de deux numéros sur le militantisme et le changement social reflète donc une volonté de contribuer à une réflexion sur le militantisme et sur sa place dans le champ de l'intervention sociale et communautaire, notamment en contexte francophone minoritaire. Nous estimons que le militantisme devrait non seulement être reconnu comme une pratique légitime et pertinente, mais également occuper une place beaucoup plus importante dans la formation et la pratique du travail social.

\section{Contenu et structure du numéro}

Le numéro s'amorce avec une entrevue accordée par une personne activiste au sein du mouvement Black Lives Matter Toronto, qui témoigne notamment de ses réalités en lien avec le racisme et la brutalité policière. Ses propos mettent en lumière la nécessité de militer pour la reconnaissance des personnes noires et pour le respect de leurs droits, particulièrement dans le contexte actuel où le racisme constitue un problème systémique dans les services policiers et dans plusieurs autres sphères de notre société.

Six articles composent Le dossier, abordant des dimensions théoriques et pratiques en lien avec le militantisme et le changement social. Dans le premier article, Christian Jetté se penche sur la place du militantisme au sein de l'action collective et communautaire. Pour ce faire, il dresse un portrait de l'histoire et du développement de l'action collective dans les Amériques et en Europe, s’intéressant particulièrement aux liens étroits entre l'action collective et les questions sociopolitiques. Jetté examine également les trajectoires de militantes et militants, de pionnières et pionniers, et de certaines des figures les plus marquantes de l'intervention collective, ainsi que les principes et les valeurs ayant guidé leurs réflexions. L'auteur termine l'article par une analyse de l'action communautaire à la lumière de la théorie de la reconnaissance de Honneth.

Dans le deuxième article, Méric Sauvé et Ysabel Provencher examinent, à partir d'auteurs canadiens et américains, les pratiques orchestrées par les organismes et les groupes communautaires en matière de défense collective des droits. Les auteurs proposent ensuite une classification plus fine de ces pratiques, ces dernières étant modélisées autour de trois grandes stratégies : les stratégies émancipatoires, les stratégies de coopération-persuasion et les stratégies de confrontation. La classification de Sauvé et Provencher se veut un regard actualisé sur les pratiques communautaires en matière de défense collective des droits, permettant ainsi de mieux les (re)connaître et, possiblement, les améliorer. 
Le troisième article, rédigé par Maria Nengeh Mensah, Janik Bastien Charlebois, Olivier Vallerand, Sandra Wesley et Ken Monteith, se penche sur le militantisme par l'entremise des témoignages de personnes qui luttent pour l'inclusion sociale de leurs communautés sexuelles et de genres. Il présente les résultats d'une recherche fondée sur le témoignage de vingt militantes et militants issus de trois groupes sociaux : les personnes lesbiennes, gaies, bisexuelles, trans, queer et intersexes (LGBTQI), les personnes vivant avec le VIH et les personnes ayant une expérience de travail du sexe. Les auteurs mettent de l'avant une conception politique, sensible et intersectionnelle de la notion de communauté afin de dresser un portrait transversal des expériences de militance par le témoignage tout en relevant les singularités des points de vue qui la composent.

Dans le quatrième article, Lise Savoie, Marie-Andrée Pelland, Hélène Albert et Isabel Lanteigne présentent les résultats d'une recherche-action menée en 2014 auprès de femmes du secteur communautaire. Dans l'objectif de saisir les trajectoires d'engagement de ces femmes, les auteures s'intéressent plus spécifiquement aux motifs d'engagement passés et actuels, ainsi qu'au sens qu'elles accordent à leur engagement. S'appuyant sur des récits de vie, les auteures mettent en lumière l'imbrication de l'engagement professionnel et de l'engagement personnel dans les trajectoires des femmes, où se posent les questions de justice sociale, d'entraide et de solidarité et le souci de reconstruire un monde équitable.

Dans le cinquième article, Marianne Chbat rend compte des diverses façons dont certaines personnes LGBTQ+ d'origine libanaise articulent et négocient leur identification ethno-sexuelle. À partir d'une lunette intersectionnelle, cette recherche suggère qu'il existe une multiplicité de discours identitaires, ces derniers déstabilisant parfois, ou reproduisant tantôt, les représentations qui sont associées à une identité homosexuelle " hégémonique » ou, autrement dit, une identité homosexuelle forgée autour de certains stéréotypes et de représentations rigides/normatives entourant « la bonne manière » de vivre son homosexualité dans la société occidentale.

Enfin, dans le sixième article, Roxanne Lorrain explore le sens qu'attribuent les usagères à leur participation auprès de comités de parents dans plusieurs maisons de naissance. En combinant les méthodes qualitatives à une fine analyse sociohistorique des maisons de naissance québécoises, la chercheure montre comment les rapports entre les femmes, les sages-femmes et les maisons de naissance ont progressivement mené à une certaine perte de reconnaissance des usagères au sein de ces organisations. Des pistes novatrices pour le militantisme et le travail social sont abordées en conclusion.

Le numéro examine ensuite des pratiques novatrices dans la section Des pratiques à notre image. Le premier texte examine la place qu'occupent les jeunes au sein du 
militantisme, plus spécifiquement la place d'un jeune élève qui milite au sein du mouvement de jeunesse franco-ontarienne, tandis que le deuxième texte porte sur la défense de droits et le militantisme en maison d'hébergement pour femmes victimes de violence conjugale.

Bonne lecture!

\section{Bibliographie}

BOCK, Michel (2010). "Quelle histoire nationale pour les minorités canadiennes-françaises? », dans Éric Bédard et Serge Cantin (dirs.), L'Histoire nationale en débat. Regards croisés sur la France et le Québec, Paris, Riveneuve Éditions, p. 115-133.

BOISCLAIR, Odile, et collab. (2010). «L'intervention féministe dans les centres de femmes : en action pour un monde plus juste! », dans Christine Corbeil et Isabelle Marchand (dirs.), Lintervention féministe d'hier à aujourd'hui : portrait d'une pratique sociale diversifiée, Éditions du remue-ménage, p. 211-227.

DAVIS, Angela (1997). Frontline: Interview with Angela Davis, réseau PBS, réf. du 31 mars 2017, http://www.pbs.org/wgbh/pages/frontline/shows/race/interviews/davis.html

LAPIERRE, Simon, et Joscelyne LEVESQUE (2013). « 25 ans plus tard... et toujours nécessaires! Les approches structurelles dans le champ de l'intervention sociale », Reflets: revue d'intervention sociale et communautaire, Vol. 19, № 1, p. 38-64.

MUlLALY, Bob (2007). The New Structural Social Work, Third Edition, Oxford University Press, $398 \mathrm{p}$.

PALUMBO, Emma, et May FRIEDMAN (2014). « Occupying Social Work: Unpacking Connections and Contradictions in the Social Work/Activist Divide ", CAOS: The Journal of Critical AntiOppressive Social Inquiry, Vol. 1, p. 82-100.

PULLEN-SANSFAÇON, Annie (2013). "La pratique anti-oppressive ", dans Henri Dorvil et Elizabeth Harper (dirs.), Le travail social : théories, méthodologies et pratiques, Québec, Presses de l'Université du Québec, p. 353-373.

ST-AMAND, Nérée (2010). Repenser le service social?, Ottawa, Merriam Print, 324 p. 